an, und es wird das Mannit unverändert wieder erhalten, nachdem er dadurch zersetzt ist. Der dabei gebildete kohlensaure Baryt ist wasserfrei.

Der Mannitbaryt verträgt einen höheren Hitzgrad als der Rohrzuckerbaryt, ehe er dadurch zersetzt wird. Dabei wird er erst ziegelroth wie der Mannitkalk, stärker erhitzt bläht er sich auf und verglimmt sehr schnell, indem er einen Haufen Asche zurüicklärst. Der Dampf, welcher dabei entsteht, riecht wie der, welcher bei den andern durch Hitze zersetzten Mannitverbindungen sich entwickelt, und brennt gleichfalls mit heller leuchtender Flamme.

Behufs der längeren Einwirkung der Alkalien auf Mannit wurden Kali, Natron, Kalk und Baryt mit in Wasser gelöstem Mannit zusammen fast ein Jahr lang digerirt, und zwar in dem Verhältnisse und der Art, wie die Digestionsversuche mit dem Rohrzucker vorgenommen waren. Nämlich theils mit Ueberschufs von Kalk und Baryt, theils die kalt und theils die heifs gesättigten klaren Lösungen. Nur die Quantitäten der Versuchsflüssigkeiten waren weniger, als bei den Versuchen mit Rohrzucker, indem dieselben nur den vierten Theil betrugen.

Um nun die etwaigen Veränderungen, welche durch die Digestion mit den Alkalien das Mannit erfahren haben konnte, zu erfurschen, wurden dieselben Prüfungen mit den Versuchsflüssigkeiten angestellt, wie sie beim Rohrzucker beschrieben sind.

Alle Versuche ergaben aber (was wohl überflüssig ist detaillirt anzuführen), dafs das Mannit unverändert geblieben war.

\title{
Einige Notizen und Erfahrungen über die inländische Zuckerfabrikation;
} von

\author{
Dr. L. F. Bley \\ in Bernburg.
}

Nachdem vor fast 100 Jahren durch den A potheker Marg g raf in Berlin der Zuckergehalt der Runkelrüben zuerst nachgewiesen worden war, fing man am Ende des 18ten Jahrhunderts in Deutschland zuerst an Arch. d.Pharm. II. Reihe. XXIX. Bds. I. Hft. 
der fabrikmä fsigen Darstellung dieses inländischen Zukkers einige Aufmerksamkeit zu schenken und Professor Lampadius in Freiberg war der erste, welcher eine Fabrik anlegte. Er gewann inders damals nur 2, bis 2,5 $\frac{0}{0}$ Rohzucker. Achard und Hermbstädt in Berlin sahen bessere Resultate, indem sie bis $5,5 \div$ Zucker erhielten. J. B. Trommsdorff in Erfurt und das Nationalinstitut, so wie Parmentier in Paris hielten, auf Versuche gestiitzt, die Fabriliation des Rübenzuckers nicht für vortheilhaft, da die Kusten gegen die geringe Ausbeute sich zu hoch stellten. Die französische Politik nnter Napoleon legte der Einfuhr des Colonialzuckers hohe Steuern auf und begiinstigte dadurch die inländische Fabrikation bedeutend. Nicht allein in Frankreich entstanden melure Fabriken, sondern auch in Norddeutschland, die in Krayn in Schlesien, in Althaldensleben und Quedlinburg. In Frankreich sollten im Jahre 1812 viele Musterfabriken auf kaiserlichen Befehl angelegt und die Fabrikation in möglichst grofsartigen Betrieb gesetzt werden, als die für jenes Land und seine damalige Regierung unglückliche Katastrophe cintrat, wodurch diese Angelegenheit ins Stocken gerieth, doch hielten sich einzelne Fabriken, als jene zu Arras, anch unter den ungünstigsten Umständen noch mehre Jahre lang, indem sie bekanntlich einer bessern Zukunft entgegen sahen. Auch die letztgenannten deutschen Fabriken arbeiteten selbst nach geschlossenem Frieden und dadurch sehr gesunkenen Zuckerpreisen noch mehre Jahre fort, bis sie etwa im Jahre 1818 ihre Arbeit einstellten.

Dubrunfant gab im Jahre 1825 ein neues Werk, denen bald mehre andere folgten, über Darstellung des Rübenzuckers heraus, welches in Frankreich diesem Fabrikzweige wieder neue Gunst zuwendete. Schnell hob sich dort und in Belgien derselbe, es entstanden neue Fabrikanlagen, und während die französischen Fabriken im Jahre 1829 nur 8 Millionen Pfund Rübenzucker darstellten, wurde 6 Jahre später schon das Zelınfache gewonnen. Dieses schnelle Emporblühen liefs das damalige 
Ministerium Nachtheile für die Zucker liefernden Colonien fürchten, so dafs es eine Besteuerung des Rübenzuckers vorschlug. Nach den günstigen Vorgängen der französischen Fabriken und der dadurch sehr gestiegenen Rente des Rüben erzeugenden Bodens, begann auch in Deutschland dieser Gewerbszweig wieder neu zu entstehen, und in Böhmen, Oestreich, Mähren, Ungarn, Sachsen, Preufsen, Baiern, Hannover, Würtemberg, Baden, Hessen und Anhalt entstanden vicle zum Theil grofsartige Fabriken. Auch Rufsland wandte dem neuen Fabrikationszweige seine Gunst zu, und gerade dort macht derselbe sehr gute Fortschritte. Während in Oestreich die Fabriken durch höhere Besteurung des Colonialzuckers begünstigt wurden, fing in den deutschen Zollvereinsstaaten der Ausfall für die verminderte Einfuhr an Bedenken zu erregen, und kaum, zum Theil noch nicht einmal, hatten die neuen Fabriken die darauf verwendeten ansehnlichen Kapitalien einigermafsen gesichert durch günstige Resultate, als eine weit geringere Besteurung eingeführt ward. Die Fabriken arbeiteten fort, man legte hier und da noch neue an, nicht nur mit gehöriger Prüfung über die Tauglichkeit des Bodens zum Rübenbau, sondern auch über die vorhandliche und erforderliche Menge der Arbeiter, als die Vergünstigung gegen Holland zur Einführung seines Colonial. zuckers eintrat, welche noch durch die von der holländischen Regierung auf die Ausfuhr gelegten Prämien um so drückender für die inländischen Fabriken wurde. Schon hatte die Landwirthschaft in denjenigen Ländern, deren Boden der Rübenerzeugung günstig war, eine ansehnliche Erhöhung der Bodenrente erfahren, als diese Neurungsmafsregel nothwendig einen Wendepunct für die inländische Zuckerfabrikation begründen mufste. Der Bedarf an Zucker betrug während den letzten Jahren in Deutschland jährlich etwa 1,100,000 Centner, die Menge des daselbst erzeugten Rohzuckers aber höchstens 300,000 Ctr., mithin blieben der Einfuhr noch 800,000 Ctr. Der deutsche Rübenzucker kostet den Fabriken im 
Durchschnitte etwa 10 Rthlr. der Cent., der holländische Zucker kann aber für 8 höclistens 9Rthlr. in dieV ereinsstaaten gelangen. Hierdurch werden nothwendig die Fabriken, wo nicht zu Grunde gerichtet, doch einer kläglichen Zukunft entgegen geführt, welches um so eher bei denjenigen der Fall sein wird, wo der Boden der Rübenerzeugung minder günstig, wo der Ackerzins, das Arbeitslohn und der Preis des Feuermaterials sich hochstellt, und wo man weniger sorgfältig bei der Cultur der Rüben und der Fabrikation des Zuckers zu Werke geht.

Es wäre aber sehr zu bedauren, wenn der neue wichtige Erwerbszweig für Deutschland, welchen die Darstellung des Rübenzuckers ausmacht, wieder verloren gehen sollte, weil derselbe manche sehr beachtenswerthe Vortheile gewährt. Denn erstens giebt er der Landwirthschaft eine sehr ansehnliche Rente, indem bei uns der Magdeburger Morgen einen Reinertrag von $16 \mathrm{Rth} / \mathrm{r}$. giebt. Zweitens gewährt er einer grofsen Anzahl Menschenhänden Arbeit, in einer Zeit, wo diese, wie in den Monaten der Campagne, als von der Mitte des Septembers bis Mitte Februars in vielen Gegenden fehlt. Auch die Cultur beschäftigt viele Arbeiter, da die Spatenkultur grofse Vortheile vor der mittelst des Pfluges zeigt. Hierdurch aber trägt derselbe zur grörsern Wohlhabenheit der Gegend bei.

Die Furcht, durch die gröfsere Ausbreitung der inländischen Zuckerfabriken am Handel mit den Zucker erzeugenden Ländern aufserhalb Europa zu verlieren, ist wohl deshalb ungegründet, weil Deutschland von daher viel mehr bezieht, als es dahin ausführt und selbst, wenn es seinen ganzen Zuckerbedarf im Lande gewönne, dennoch ein ansehnlicher Ueberschufs der Einfuhr jener ausländischen Producte gegen die Einfuhr von deutschen Producten und Waaren bleiben wïrde. Wenn es also sehr wünschenswerth scheint, dafs die inländische Zuckerfabrikation unter dem Schutze zweckmäfsiger Gesetze sich noch mehr heben mürste, so scheint es mir nicht überflüssig auf einige Erfahrungen aufmerksam zu 
machen, welche diesem Gewerbszweige Nutzen gewähren können, und welche ich bei Untersuchung diesjähriger Zuckerrïben und daraus gewonnenem Fabrikate gemacht habe.

In den diesjährigen Runkelrüben fand ich alle Bestandtheile, welche Pelouze darin nachgewiesen hat, nämlich: krystallinischen Zucker, Aepfelsäure, Kleesänre, Eiweirs, Gallert, Ferment, ätherisches und fettes Pflanzenöl, Chlorophyll, Gummi, Faserstoff, schwefelsaures und kleesaures Kali, salzsaures und kleesaures Ammoniak, kleesauren Kalk, Thonerde, Eisen und Manganoxyd, Schwefel und Wasser, aufserdem aber noch salpetersaures Kali.

Die Menge des Zuckers beträgt in diesjährigen Rüben hiesiger Gegend bis zu 3 Pfund. Schwere 9,50 $\frac{0}{0}$, in den gröfsern nur 7,25 $\frac{0}{0}$, obschon letztere ansehnlich mehr Saft liefern.

Die gröfsern Rüben erhalten einen ansehnlichen Ueberschufs an Gummi und Gallerte. In denselben ist eine ammoniakalisch bittre Substanz enthalten, welche dem aus diesen Rüben gewonnenen Zucker einen geringern Werth giebt.

Diese Substanz hat sich wahrscheinlich durch Einflufs der nicht verwesenden Düngstoffe in den Rüben erzeugt. Sie hat ihren Sitz mehr am Kopfe der Rübe, daher ein weiteres Abschneiden des oberen Theiles rathsam scheint.

Auffallend ist es, dafs der Läuterungsprocefs bei dem Safte der gröfsern Rüben schwieriger, als bei dem Safte der kleinern (von $\frac{1}{2}-3 \mathbf{P f}$.) ist, auch bei wesentlich vermehrtem Kalkzusatze, bei erstern dennoch nicht so gut gelingt, als bei letztern. Der Zucker aus den grö̈sern Rüben ist daher auch mehr bräunlich gefärbt, als der der kleinern.

Die Reinigung des zu stark mit Kalk versetzten Zuckers gelingt recht gut durch Zusatz von saurem phosphorsaurem Kalk, bis zum geringen Vorwalten des Kalkes, welches man mittelst geröthetem Lackmuspapiere, 
welches aber noch blä口lich gefärbt werden mufs, erkennt.

Die Reinigung des schmierigen Zuckers, der viel Kalk, Gummi, Farbstoff und salpetersaure Salze enthält, gelingt am Besten durch Auflïsen in Wasser, Zusatz von saurem phosphorsauren Kalk bis zur starken Säurung, warmgestellt mit frisch ausgeglühetem Holzkohlenpulver, nicht mit Thierkohle, Sättigen des filtrirten Saftes mit Kalkmilch, abermaligem Filtriren und Eindampfung.

Auf gleiche Weise gelingt die Reinigung der geringen Melasse, bei der zuvor Aufkochen mit Eiweifs oder Blut zweckmäfsig ist.

Ein sehr gutes Reinigungsmittel von Farbstoff ist das Thonerdehydrat, welches man darstellt, indem man Alaun in Wasser löset und so lange kohlensaures $\mathrm{Na}$ trum zusetzt, bis noch ein weilser Niederschlag erfolgt, den man auf einem Filtrirbeutel sammelt und aussüfst. Wegen sciner Kostspieligkeit aber eignet es sich mehr zu Versuchen im Kleinen, als zu fabrikmärsiger Verwendung.

Für die Fabriken würde es nützlich sein, zuweilen Proben mit den Rüben auf Zuckergehalt anzustellen, was nach meiner Methode am Besten so geschieht, dafs man den fein geriebenen Rübenbrei mit starkem Weingeist von $90 \frac{0}{0}$ übergiefst, in gelinder Wärme einige Stunden stehen läfst, öfters umschüttelt, dann ausprefst, der Flüssigkeit ein wenig Kalkhydrat zusetzt, aufkocht, filtrirt und abdunstet, den Zucker aber zwischen Filtrirpapier trocknet und wägt, wodurch man schnell den Zuckergehalt nachweisen kann.

In Hinsicht der Cultur der Rüben möchte es vortheilhaft für die Fabriken sein, den Landwirthen die Anwendung des frisch gedüngten Ackers zum Rübenbau zu untersagen.

Ebenso würde es für die Fabriken nützlich sein, die kleinern Rüben von $\frac{1}{2} \mathbf{- 3}$ Pfund Schwere den gröfsern 
vorzuziehen und die letztern mit geringern Preisen zu bezahlen."

Sodann möchte es in solchen Jahren wie das vorige, wo das erste Frühjahr trocken und warm ist, späterhin aber feuchte Witterung eintritt, gut sein, die Rübenerndte nicht vor Mitte September, wo möglich aber noch später zu beginnen.

Hinsichtlich des Reinigungsprocesses des Rohzuckers kann den Fabriken nicht genug die gröfste Sorgfalt beim Auswaschen der schon gebrauchten Kohle empfohlen werden, da beim Unterlassen dieser Malsregel eine Menge von Unannehmlichkeiten für die Fabrikanten eines Zuckerbereitungsprocesses entstehen, deren nachherige Beseitigung schwierig und kostspielig ist.

\section{Ueber Fremy's Eisensäure.}

Mein Freund und Schwager H. Trommsdorff in Erfurt, welcher durch Privatmittheilung Kenntnifs von der Entdeckung Fremy's über die Eisensäure erhielt, theilt mir darüber Folgendes mit:

Mit der Säure selbst ist der Entdecker nuch beschäftigt.

Eisensaures Kali erhielt Tromm sd or f auf folgende Weise :

Er mengte 2 Drachmen sehr fein gepulverte Eisenfeile mit 4 Drachmen gepulverten salpeters. Kali, trug dieses Gemenge in einen schwach dunkelroth glühenden Tiegel von 8-10 Unzen Inhalt auf einmal ein, während der Tiegel noch inmitten glïhender Kohlen stand. Sobald die Verbindung nach Art einer Explosion, unter starker Lichtentwicklung und Ausstofsen eines weifsen Dampfes an einem Ende der Masse beginnt, nimmt man den Tiegel aus dem Feuer. Die Verpuffung verbreitet sich schnell durch die ganze Masse, und sobald sie aufgehört hat, stöfst man mit Hülfe eines eisernen Spatels die Masse aus dem Tiegel anf ein kaltes Blech. 